\title{
Die Anrechnung non-formalen und informellen Lernens auf Hochschulstudiengänge in Deutschland
}

\author{
Wolfgang Müskens • Sonja Lübben
}

Eingegangen: 31. Mai 2018 / Angenommen: 6. September 2018 / Online publiziert: 21. September 2018 (C) Der/die Autor(en) 2018

Zusammenfassung Bei der Anrechnung außerhochschulischer Vorleistungen auf Hochschulstudiengänge wird häufig von „,beruflichen Kompetenzen“ gesprochen. Die Ergebnisse der AnHoSt-Studie von 2014 sowie des Anrechnungsmonitors 2016 zeigen jedoch, dass sich die Anrechnungsverfahren an deutschen Hochschulen bislang überwiegend auf formelle Qualifikationen beziehen, nicht aber auf Kompetenzen, die in non-formalen oder informellen Lernkontexten erworben wurden. Selbst individuelle Anrechnungsverfahren basieren in aller Regel auf den formalen Curricula von Aus- und Fortbildungen.

International wird das Thema „Anrechnung“ häufig mit einer Anerkennung nonformal und informell erworbener Kompetenzen gleichgesetzt. Ansätze wie „APEL“ (Accreditation of Prior Experiential Learning) und „PLAR“ (Prior Learning Assessment and Recognition) konzentrieren sich auf informell erworbene Kompetenzen und beschäftigen sich mit deren Erfassung und Bewertung. Eine Betrachtung der gesetzlichen Situation sowie der nationalen Beschlüsse zur Anrechnung in Deutschland zeigt, dass sowohl die KMK-Beschlüsse zur Anrechnung als auch die entsprechende Umsetzung in die Landeshochschulgesetze die Berücksichtigung non-formal und informell erworbener Kompetenzen bei der Anrechnung auch hierzulande zulassen. Am Beispiel des PLAR-Services der Universität Oldenburg soll gezeigt werden, wie die hochschulweite Anrechnung non-formal und informell erworbener Kompetenzen implementiert werden kann. Dabei werden die Besonderheiten des PLAR-

\footnotetext{
Publisher's Note Springer Nature remains neutral with regard to jurisdictional claims in published maps and institutional affiliations.

Dr. W. Müskens $(\bowtie) \cdot$ Dipl.-Päd. S. Lübben

Fakultät I - Bildungs- und Sozialwissenschaften, Institut für Pädagogik, Kompetenzbereich

Anrechnung, Carl von Ossietzky Universität Oldenburg, 26111 Oldenburg, Deutschland

E-Mail: wolfgang.mueskens@uni-oldenburg.de

Dipl.-Päd. S. Lübben

E-Mail: sonja.luebben@uni-oldenburg.de
} 
Ansatzes dargestellt, der insbesondere die Berücksichtigung informell erworbener Kompetenzen unterstützt.

Schlüsselwörter Anrechnung · Informell erworbene Kompetenzen · Niveaubestimmung · Prior Learning Assessment and Recognition (PLAR) · Portfolio

\section{The crediting of non-formal and informal learning on higher education courses in Germany}

Abstract When crediting non-university previous achievements in higher education courses, the term "professional competence" is often used. However, the results of the 2014 "AnHoSt study" and the 2016 "Anrechnungsmonitor" show that the crediting procedures at German HEIs have so far mainly related to formal qualifications, but not to competences acquired in non-formal or informal learning contexts. Even individual crediting procedures are generally based on the formal curricula of apprenticeship and further training.

Internationally, the topic of "crediting" is often equated with the recognition of non-formally and informally acquired competences. Approaches such as "APEL" (Accreditation of Prior Experiential Learning) and "PLAR" (Prior Learning Assessment and Recognition) focus on informally acquired competences and deal with their valid recording and validation. An examination of the legal situation and the national resolutions on crediting in Germany shows that both the KMK resolutions on crediting and the corresponding implementation in the state university laws permit nonformally and informally acquired competences to be taken into account in crediting also in this country. The PLAR service of the University of Oldenburg will be used as an example to show how non-formal and informal crediting of competences at universities can be implemented. The special features of the PLAR approach, which particularly supports the consideration of informally acquired competences, are being described.

Keywords Recognition of Prior Learning (RPL) · Informally acquired competences · Level Assessment · Prior Learning Assessment and Recognition $($ PLAR) $\cdot$ Portfolio

\section{Einleitung}

Derzeit kommt den Möglichkeiten zur Anerkennung bzw. Anrechnung bereits erworbener Kompetenzen, Lernergebnisse und -erfahrungen auf Aktivitäten der weiteren Berufsbiografie deutschlandweit eine stetig wachsende Bedeutung zu. Die Wichtigkeit vom Lernen im Lebenslauf, die Bedeutung von außerhalb des formalen Bildungswesens angeeigneten Kompetenzen und damit Möglichkeiten der Anrechnung non-formaler und informeller Lernleistungen geraten nicht nur im betrieblichen Kontext, sondern auch im Hochschulbereich zunehmend in den Blick. Mittlerweile nehmen Hochschulen bundesweit die Unterstützung lebenslangen Lernens und die 
Sicherstellung von Durchlässigkeit zwischen beruflicher und hochschulischer Bildung als eine bedeutende politische und gesellschaftliche Aufgabe wahr. Auch die Diskussion um die Anerkennung von Kompetenzen, die in non-formalen oder informellen Lernkontexten erworben wurden, findet inzwischen Eingang in die Hochschulgesetze der Länder (Schreiber 2009; Annen 2012; Werquin 2015).

Als Gründe für diese Entwicklung werden der demografische Wandel, der technische Fortschritt, ein steigender Bedarf an hochqualifizierten Fachkräften, die Schaffung eines gemeinsamen europäischen Wirtschafts- und Wissenschaftsraums sowie die Bedeutung akademischer Bildung für den Erhalt und Ausbau des wirtschaftlichen und gesellschaftlichen Wohlstands Deutschlands gesehen.

Während die Möglichkeiten der Anrechnung von Kompetenzen, die in non-formalen oder informellen Lernkontexten erworben wurden, in Deutschland erst seit jüngerer Zeit diskutiert werden, bildet diese Thematik im internationalen Diskurs bereits seit vielen Jahrzehnten einen festen Bestandteil (erziehungs-)wissenschaftlicher sowie bildungspolitischer Debatten. So werden die Begriffe informal education und informal learning bereits seit den 1950er-Jahren in der amerikanischen, der britischen und der französischen Erwachsenenbildung diskutiert. Entsprechend können diese Länder mittlerweile auf eine langjährige Erfahrung mit Verfahren zur Erfassung und Anrechnung außerhalb formaler Bildungssysteme erlangter Kompetenzen zurückblicken (Dohmen 2001).

In der politischen Diskussion in Deutschland ist die Frage der Anrechnung von Lernergebnissen aus non-formalen oder informellen Lernkontexten gegenwärtig stark auf den Bereich der beruflichen Bildung (als Ziel der Anrechnung) fokussiert (vgl. BMBF 2018). In dem hier vorliegenden Beitrag soll der Frage nachgegangen werden, inwieweit eine Anrechnung von Lernergebnissen informellen und nonformellen Lernens auch im Hochschulsektor möglich ist. Neben einer kurzen Darstellung der rechtlichen Situation soll der Frage nachgegangen werden, ob und wie Anrechnung unter den Rahmenbedingungen deutscher Hochschulen realisiert werden kann. Anhand eines Fallbeispiels an der Carl von Ossietzky Universität Oldenburg soll die Implementierung der Anrechnung beleuchtet werden.

\section{Möglichkeiten der Anrechnung von Kompetenzen im internationalen Diskurs}

In den USA werden Anerkennungs- bzw. Anrechnungsverfahren informell erworbener Kompetenzen bereits seit Ende des Zweiten Weltkriegs in größerem Umfang angewendet. Die Einführung des sogenannten GI-Bill ermöglichte den aus dem Einsatz zurückkehrenden Soldaten durch Nachweise ihrer im Krieg erworbenen Kompetenzen die Aufnahme eines Studiums sowie teilweise die Verkürzung der Studienzeit. Seither konnten sich Programme und Organisationen, die sich mit der Anerkennung und Anrechnung von informellen Kompetenzen mit Bezug zu Hochschulen beschäftigen, zunehmend etablieren (Colardyn 1994, S. 663; zit. n. Dohmen 2001, S. 94 ff.). Unter den Begriffen Prior Learning Assessment (PLA) und Prior Learning Assessment and Recognition (PLAR) sind mittlerweile eine Vielzahl an Anerkennungsverfahren zusammengefasst, die oftmals durch den Council of Adult 
and Experiental Learning (CAEL), einer nationalen Non-Profit-Organisation, die auf allen Ebenen des Hochschulwesens sowie des öffentlichen und des privaten Sektors tätig ist, um den Menschen den Zugang zu Bildung und Ausbildung zu erleichtern, betreut werden. Außerdem bietet das National Occupational Competency Testing Institute, ein in den USA führender Anbieter beruflicher Leistungstests, mehr als 170 standardisierte technische Tests für eine Vielzahl unterschiedlicher Berufe und Beschäftigungsfelder an, die berufliche Kompetenzen, Kenntnisse und Fähigkeiten überprüfen und zertifizieren (Hanft und Müskens 2003; Müskens 2007).

In Europa wurden seit den 1980er-Jahren Ansätze der Identifizierung von (informell erworbenen) Kompetenzen aus dem nordamerikanischen Raum zunehmend adaptiert. Hinsichtlich der Öffnung der Universitäten sowie der Anerkennung und Anrechnung früheren Lernens gehört Großbritannien hier zu den Pionieren. Hervorzuheben sind hier die sogenannten Accreditation of Prior Learning-Systeme (APLSysteme) an englischen Hochschulen: APL for access verfolgt das Ziel, durch den Nachweis früherer Lernerfahrungen auch ohne entsprechendem Schulabschluss eine Zugangsberechtigung zu einem Hochschulstudium zu erhalten. Bei APL for advanced standing kann der Nachweis bereits erworbener Lernergebnisse dazu führen, dass der/die Bewerber/in in ein fortgeschrittenes Semester eines Hochschulstudiengangs eingestuft wird (Hannken-Illjes und Lischka 2004; Räbinger et al. 2008). Differenziert wird zudem zwischen der jeweiligen Formalität der vorangegangenen Lernprozesse. Im Hinblick auf die Anerkennung bzw. Anrechnung bereits erworbener Qualifikationen und Kompetenzen wird hier unterschieden zwischen Accreditation of Prior Experiential Learning (APEL) und Accreditation of Prior Certificated Learning (APCL). APEL bezeichnet die Anerkennung bzw. Anrechnung bereits erworbener, jedoch bisher nicht zertifizierter Lernergebnisse und richtet den Blick auf Kenntnisse, die auf informellen Wegen (z. B. durch beiläufige Erfahrungen im Berufsleben, im Alltag oder durch Selbststudium) erlangt wurden. Mithilfe der APELSysteme durchlaufen die Bewerberinnen und Bewerber Beratungs- und Evaluationsverfahren, anhand derer sie eine Zugangsberechtigung erhalten können, selbst wenn sie nicht über die formal notwendigen Schulabschlüsse verfügen. APEL unterstützt die Kompetenzerfassung gerade in der Studieneingangsphase und reagiert auf die speziellen Bedarfe von Studierenden mit Berufs- und Lebenserfahrung. Durch APEL-Verfahren können Studienanforderungen unter Umständen reduziert werden, sofern entsprechende, gleichwertige Kompetenzen bereits in vorherigen Lernprozessen erworben wurden. Die APEL-Verfahren werden vor allem in geistes- und sozialwissenschaftlichen Fächern angewendet und sind im Hinblick auf die Öffnung der Hochschulen für Berufstätige von großer Bedeutung. APCL dagegen ist auf die Anerkennung bzw. Anrechnung von Lernergebnissen gerichtet, die in formalisierten Bildungsgängen (z. B. Weiterbildungsprogrammen) erworben wurden und in der Regel mit einem Zertifikat oder einer Bescheinigung abschließen. Somit stellt APCL ein Verfahren zur Anerkennung bzw. Anrechnung von in der Vergangenheit bereits zertifizierten Kenntnissen dar (Dohmen 2001; Zürcher 2007; Räbinger et al. 2008).

Auch in Frankreich wurden seit Mitte der 1980er-Jahre Gesetze und Richtlinien zur Anrechnung informellen Lernens an Hochschulen eingeführt. Mittlerweile hebt sich das französische System der Anrechnung und Anerkennung international von anderen Ansätzen dadurch ab, dass neben der Anrechnung von Teilen einer Quali- 
fikation auch die Verleihung gesamter Abschlüsse (einschließlich Hochschulgrade) auf der Grundlage der Anrechnung formaler, non-formaler und informeller Vorleistungen möglich sind: ,The accreditation system introduced in France in 2002 has practically no equivalent in Europe or the world. ... only the French system offers the possibility of acquiring a qualification, full or partial, without prior instruction“" (Triby 2009, S. 114). Das französische System besteht aus drei unterschiedlichen Verfahren, die jeweils gesondert gesetzlich geregelt wurden. Die Validation des études supérieures (VES) bezieht sich vorrangig auf die Anerkennung hochschulischer Vorleistungen. Die Validation des acquis professionnels (VAP) ermöglicht eine Anerkennung und Anrechnung beruflicher Fertigkeiten sowie eine direkte Aufnahme in einen Studiengang nach erfolgreicher Anerkennung vorheriger Leistungen. Das Verfahren wird von den Universitäten insbesondere bei ausländischen Studierenden angewandt, die sich an einer französischen Hochschule einschreiben wollen. Es hat den Zweck, das Niveau der vorherigen Ausbildung eines/einer Studierenden im Hinblick auf das französische System zu bestimmen und ggf. anerkennen zu lassen. Im Jahr 2002 rückte mit der Einführung von acquis de l'expérience (VAE) außerdem die Anrechnung von auf informellem Wege erworbenen Lernleistungen, Kompetenzen, Kenntnissen und Fähigkeiten sowie Erfahrungslernen (z. B. ehrenamtliche und familiäre Tätigkeiten) in den Blick. Zum Einsatz kommen u. a. Prüfungen und PortfolioAnalysen sowie Gespräche mit einer Prüfungskommission. Ziel des Verfahrens ist es, insbesondere berufliche Erfahrungen und Leistungen anerkennen zu lassen, um einen Abschluss oder ein bestimmtes Qualifikationsniveau zu erlangen. Bis heute werden die beiden Verfahren VAE und VAP zur Anerkennung bzw. Anrechnung bereits erworbener Kompetenzen auf Hochschulstudiengänge genutzt. Allerdings fällt ihre Umsetzung an den Hochschulen trotz der gesetzlichen Rahmenregelungen sehr unterschiedlich aus. Die meisten der Verfahren betreffen den Zugang zur Hochschule, nur wenige die Anrechnung von Vorkenntnissen (Greco 2002; Zürcher 2007; Campus France 2016).

Die Entwicklungen in Großbritannien und Frankreich weckten auch in anderen Teilen Europas das Interesse am Thema Anrechnung, beispielsweise in den skandinavischen Ländern, in Irland und in den Niederlanden. Als Einrichtung der Europäischen Union (EU) unterstützte insbesondere das Europäische Zentrum für die Förderung der Berufsbildung (CEDEFOP) die Beschäftigung mit der Identifizierung, Bewertung und Anerkennung informell und non-formal erworbener Kompetenzen (Björnavåld 1997; Perker und Ward 1994).

In Deutschland rückte die Diskussion um die Anerkennung bzw. Anrechnung von außerhalb der formalen (Berufs-)Bildung erworbener Lernleistungen auf Hochschulstudiengänge zwar erst vergleichsweise spät ins Blickfeld. Mittlerweile kommt der Unterstützung lebenslangen Lernens sowie den Möglichkeiten von Durchlässigkeit und Anrechnung bereits erworbener Kompetenzen jedoch auch hierzulande eine wachsende Bedeutung zu. Der Fokus liegt dabei ausdrücklich auch auf der Berücksichtigung von auf non-formalem und auf informellem Wege erworbenen Kompetenzen, Qualifikationen, Lernergebnissen und -erfahrungen. 


\section{Gesetzliche Regelungen zur Anrechnung außerhochschulisch erworbener Kompetenzen auf ein Hochschulstudium in Deutschland}

Die wesentlichen Rechte zur gesetzlichen Umsetzung von Verfahren zur Anrechnung von Kompetenzen auf Hochschulstudiengänge liegen auf der Länderebene. Auf der Bundesebene sind die rechtlichen Rahmenbedingungen zum Thema Anrechnung außerhochschulisch erworbener Kompetenzen, Kenntnisse, Fertigkeiten, Qualifikationen oder Lernergebnisse in verschiedenen Beschlüssen, Gesetzen und Verordnungen verankert und betreffen neben den Lernergebnissen formeller Qualifikationen auch Kompetenzen, die auf non-formalen bzw. auf informellem Wege erworben wurden. Insbesondere die in den Jahren 2002 und 2008 in den Beschlüssen der Kultusministerkonferenz (KMK) formulierten Empfehlungen bilden die Grundlage für die Umsetzung der Anrechnung außerhochschulisch erworbener Kompetenzen an deutschen Hochschulen (Hanft et al. 2014; Hanak und Sturm 2015).

Die erste dieser beiden KMK-Empfehlungen zur „Anrechnung von außerhalb des Hochschulwesens erworbenen Kenntnissen und Fähigkeiten auf ein Hochschulstudium“ aus dem Jahr 2002 sieht u. a. folgendes vor:

1. Außerhalb des Hochschulwesens erworbene Kenntnisse und Fähigkeiten können im Rahmen einer - ggf. auch pauschalisierten - Einstufung auf ein Hochschulstudium angerechnet werden, wenn

1.1 die für den Hochschulzugang geltenden Voraussetzungen - ggf. auch über die Möglichkeiten des Hochschulzugangs für besonders qualifizierte Berufstätige - gewährleistet werden;

1.2 sie nach Inhalt und Niveau dem Teil des Studiums gleichwertig sind, der ersetzt werden soll;

1.3 entsprechend den Grundsätzen des neuen Qualitätssicherungssystems im Hochschulbereich die qualitativ-inhaltlichen Kriterien für den Ersatz von Studienleistungen durch außerhalb des Hochschulwesens erworbene Kenntnisse und Fähigkeiten im Rahmen der Akkreditierung überprüft werden.

2. Außerhalb des Hochschulwesens erworbene Kenntnisse und Fähigkeiten können höchstens

$50 \%$ eines Hochschulstudiums ersetzen ... (KMK 2002, S. 1).

Der im Jahr 2008 veröffentlichte zweite KMK-Beschluss zur Anrechnung bekräftigt die Empfehlung aus dem Jahr 2002 und enthält zusätzliche Anhaltspunkte zu den Möglichkeiten einer Anrechnung von außerhalb des Hochschulwesens erworbenen Kenntnissen und Fähigkeiten auf ein Hochschulstudium:

Die Verbesserung der Durchlässigkeit des Bildungssystems ist ... eine wesentliche Voraussetzung, um vorhandene Potentiale zu erschließen und zu fördern. Ein wichtiger Ansatzpunkt ist in diesem Zusammenhang der Übergang beruflich qualifizierter Personen in den Hochschulbereich unter Anrechnung außerhalb des Hochschulbereichs erworbener Kenntnisse und Fähigkeiten, sodass sich die Studiendauer verkürzt und damit die Schwelle zur Aufnahme eines Studiums absinkt (KMK 2008, S. 1). 
Im Jahr 2010 hat die KMK die Anrechnung außerhochschulisch erworbener Kompetenzen zudem erstmals in die Ländergemeinsamen Strukturvorgaben für die Akkreditierung von Bachelor- und Masterstudiengängen aufgenommen (KMK 2010; Hanft et al. 2014). Von großer Bedeutung ist hierbei, dass die Anrechnung außerhalb des Hochschulbereichs erworbener Kompetenzen und Fähigkeiten als eine Muss-Bestimmung formuliert ist:

1.3 Nachgewiesene gleichwertige Kompetenzen und Fähigkeiten, die außerhalb des Hochschulbereichs erworben wurden, sind bis zur Hälfte der für den Studiengang vorgesehenen Leistungspunkte anzurechnen ... (KMK 2010, S. 3).

Die jeweiligen Beschlüsse wurden in die Landeshochschulgesetze überführt. Inzwischen sind in allen Hochschulgesetzen der Bundesländer Möglichkeiten der Anrechnung verankert (Hanft et al. 2014).

Für Studierende bedeutet die Anrechnung von Kompetenzen, dass angerechnete Teile des Studiums nicht mehr studiert werden müssen und auch nicht geprüft werden. Die Entscheidung darüber, auf welche Art und in welchem Umfang außerhochschulisch erworbene Leistungen angerechnet werden, liegt jedoch in der $\mathrm{Zu}$ ständigkeit der jeweiligen Hochschule. Dies könnte einer der Gründe sein, weshalb die Implementierung entsprechender Verfahren zur Anrechnung außerhochschulisch erworbener Kompetenzen an deutschen Hochschulen bislang eher gering ausgebildet ist (Sturm und Hanak 2018). Insbesondere die Möglichkeiten der Anrechnung von auf non-formalem oder informellem Wege erworbenen Kompetenzen gestalten sich in der Hochschulpraxis als problematisch. Zwar nähern sich die deutschen Hochschulen der Thematik, hängen im internationalen Vergleich jedoch zurück (Gnahs 2003; Hanft et al. 2014; Hanak und Sturm 2015).

\section{Pauschale, individuelle und kombinierte Anrechnungsverfahren}

Die bislang an deutschen Hochschulen praktizierten Verfahren zur Anrechnung außerhalb des formalen Bildungswesens erworbener Kompetenzen unterscheiden meist zwischen pauschaler und individueller Anrechnung sowie der sogenannten kombinierten Anrechnung (Weichert 2015).

Die pauschale Anrechnung auf ein Hochschulstudium stellt ein Verfahren dar, welches es ermöglicht, im Rahmen formaler Aus-, Fort- und Weiterbildungen erlangte Kompetenzen generell und unabhängig von der jeweils antragstellenden Person auf einen Studiengang anzurechnen. Dabei erfolgt die Anrechnung pauschal, beispielsweise auf der Grundlage einer vergleichenden Analyse und Äquivalenzprüfung des entsprechenden Curriculums der Bildungseinrichtung mit dem des Studiengangs. Eine Überprüfung oder Feststellung der Kompetenzen im Einzelfall ist bei diesem Verfahren nicht erforderlich. Die Studierenden können aufgrund eines erfolgreichen Abschlusses eines außerhochschulischen Bildungsgangs bestimmte, zuvor entsprechend dem Analyseergebnis festgelegte Studieninhalte angerechnet bekommen. Das pauschale Anrechnungsverfahren wird häufig innerhalb bestehender Kooperationen zwischen Bildungseinrichtungen angewandt. Meist wird mit diesem Verfahren eine homogene Zielgruppe angesprochen, die über fachlich einschlägige Vorerfahrun- 
gen verfügt, beispielsweise eine Gruppe von Absolventinnen und Absolventen eines speziellen Lehrgangs, der als solcher angerechnet werden kann (Hanft et al. 2014; DAbeKom 2016).

Das individuelle Anrechnungsverfahren dagegen basiert auf einer individuellen Einzelfallprüfung von Unterlagen einzelner Bewerberinnen und Bewerber. Beim individuellen Anrechnungsverfahren erfolgt die Überprüfung möglicher Äquivalenzen auf der Basis von Belegen, die der/die jeweilige Studierende vorlegt. Im Idealfall wird hier ein strukturiertes Portfolio-Verfahren eingesetzt, in dem die Studierenden ihre bereits erworbenen Qualifikationen und Kompetenzen beschreiben und diese, wenn möglich, durch entsprechende Dokumente, Zertifikate, Zeugnisse o. Ä. belegen. Die Äquivalenzprüfung erfolgt - ebenso wie die daraus resultierende Anrechnungsentscheidung - für den Einzelfall. Das individuelle Verfahren wird häufig dann eingesetzt, wenn nicht oder nur zum Teil geregelte Aus-, Fort- oder Weiterbildungen absolviert oder die Kompetenzen auf non-formalen oder informellen Wegen erlangt wurden (DAbeKom 2016). Die Überprüfung von Fähigkeiten zielt darauf ab, die Kenntnisse, Fähigkeiten und Kompetenzen einer Person sowie die Eignung und Motivation zu ermitteln und den beruflichen Hintergrund $\mathrm{zu}$ analysieren, um einen weiteren Schritt in der beruflichen Laufbahn festzulegen. Die Überprüfung von Fähigkeiten soll den einzelnen Studierenden helfen, die eigene Position in der bisherigen Berufsbiografie selbst einzuschätzen, den weiteren Berufsweg zu planen oder sich auf das Anrechnungsverfahren non-formaler oder informeller Lernergebnisse vorzubereiten. Lernergebnisse sind in diesem Zusammenhang Aussagen darüber, was Lernende wissen, verstehen oder in der Lage sind zu tun, nachdem sie einen Lernprozess abgeschlossen haben (Rat der Europäischen Union 2012).

Im kombinierten Anrechnungsverfahren werden Elemente des pauschalen und des individuellen Verfahrens miteinander verbunden, um den Durchführungsaufwand möglichst gering zu halten und dennoch ein breites Spektrum von Lernergebnissen anrechnen zu können. Der Vorteil des kombinierten Anrechnungsverfahrens besteht darin, dass außerhalb der Hochschule erworbene Leistungen, die im Rahmen der Äquivalenzfeststellung im pauschalen Verfahren nicht oder nicht vollständig auf ein Studienmodul angerechnet wurden, durch ein individuelles Verfahren überprüft und ggf. angerechnet werden können, wodurch sich der Umfang der anrechenbaren Teile u. U. vergrößert (Weichert 2015).

\section{Modellprojekte zur Entwicklung und Implementierung von Anrechnungsverfahren}

Ein bedeutsames Programm zur Verankerung von Durchlässigkeit und Anrechnung in Hochschulen in Deutschland stellt die vom Bundesministerium für Bildung und Forschung (BMBF) und mit Mitteln des Europäischen Sozialfonds (ESF) geförderte Initiative Anrechnung beruflicher Kompetenzen auf Hochschulstudiengänge (ANKOM) dar, von der in drei Förderphasen in den Jahren von 2005 bis 2014 wichtige Impulse zur Implementierung von Durchlässigkeit und Anrechnung im Hochschulsystem ausgingen (Hanft et al. 2014). Insgesamt wurden 20 Projekte sowie die wissenschaftliche Begleitung gefördert. Die Aufgabe der Projekte bestand in der 
Entwicklung von Verfahren zur Anrechnung von in beruflicher Weiterbildung erworbenen Qualifikationen auf ein Hochschulstudium. Im Rahmen der Projekte untersuchten die verschiedenen Akteurinnen und Akteure der beruflichen Bildung und die der Hochschulen gemeinsam exemplarisch Gleichwertigkeiten in ausgewählten Fachbereichen und beschäftigten sich mit Aspekten und Fragestellungen hinsichtlich der Entwicklung von Äquivalenzfeststellungsverfahren. Schwerpunkte bildeten die konzeptionelle Entwicklung von Ansätzen zur Prüfung der Gleichwertigkeit hochschulischer und beruflicher Bildung, die Sichtbarmachung und Darstellung von miteinander vergleichbaren Lernergebnissen, die Entwicklung von Äquivalenzfeststellungsinstrumenten sowie die Identifizierung von Rahmenbedingungen, die die Umsetzung von Anrechnung beeinflussen (ANKOM 2016; Stamm-Riemer et al. 2008). Ziel der Initiative war es, den Übergang in ein Hochschulstudium zu verbessern und den Studierenden das erfolgreiche Absolvieren ihres Studiums neben einer Berufstätigkeit zu erleichtern. Ab 2012 wurden in einer neuen Projektphase weitere Modellprojekte gefördert, um Maßnahmen, die beruflich Qualifizierten das Studium erleichtern und einen Bezug zur Anrechnung von außerhochschulisch erworbenen Kompetenzen haben, zu implementieren und zu erproben. Die Zielgruppen der Projekte waren vor allem Studieninteressierte und Studierende, aber auch Hochschullehrende, Studienberatungen und Betriebe. Die wissenschaftliche Begleitung der ANKOM-Projekte entwickelte auf der Grundlage der Erfahrungen, die im Rahmen der ANKOM-Initiative gemacht wurden, eine Leitlinie für qualitätsgesicherte Anrechnungsverfahren im Rahmen von Hochschulstudiengängen - kurz: Anrechnungsleitlinie. Diese soll dazu beitragen, die Hochschulen bei der Anrechnung von vorangegangenen Lernergebnissen zu unterstützen. Die Anrechnungsleitlinie wurde im Januar 2010 veröffentlicht und dürfte heute zu den in der Hochschulpraxis häufig eingesetzten Informationsmaterialien zählen. Die im Rahmen von ANKOM geförderten Hochschulen gelten heute vielfach als Good-Practice-Beispiele, an denen sich andere Hochschulen orientieren (ANKOM 2010; Hanft et al. 2014).

Ein weiteres großes Förderprogramm im Hinblick auf die Anrechnung bereits erworbener Kompetenzen stellt der seit 2008 durch das Bundesministerium für Bildung und Forschung (BMBF) geförderte Bund-Länder-Wettbewerb „Aufstieg durch Bildung: offene Hochschulen“ dar. Ziel dieser Qualifizierungsinitiative ist es, die Bildungschancen aller Bürgerinnen und Bürger zu steigern und die internationale Wettbewerbsfähigkeit des Wissenschaftssystems durch nachhaltige Profilbildung im lebenslangen wissenschaftlichen Lernen und beim berufsbegleitenden Studium zu stärken. In der ersten Wettbewerbsrunde entwickelten mehr als 50 Hochschulen Programme für Berufstätige und andere Zielgruppen. Eine weitere Wettbewerbsrunde läuft seit 2014. Auch dieses Programm verfolgt das Ziel, Konzepte für berufsbegleitendes Studieren und lebenslanges wissenschaftliches Lernen besonders für Berufstätige, Personen mit Familienpflichten sowie Berufsrückkehrerinnen und Berufsrückkehrern zu fördern. Wichtige Aspekte bilden die Verbesserung der Durchlässigkeit und Verzahnung zwischen beruflicher und akademischer Bildung und die schnellere Integration neuen Wissens in die Praxis. Diese Ziele verdeutlichen die besondere Bedeutung, die dem Thema Anrechnung im Rahmen der Förderprojekte zukommt (Hanft et al. 2014; BMBF 2016). Im Rahmen des Bund-Länder-Wettbewerbs wird das Thema Anrechnung außerhochschulisch erworbener Kompetenzen 
von einer Vielzahl von Projekten auf unterschiedliche Weise aufgegriffen. Neben der Entwicklung von Verfahren der individuellen oder pauschalen Anrechnung für bestimmte Studiengänge oder spezifischer Anrechnungsrahmenordnungen stehen auch die Erprobung und Umsetzung von Anrechnungsmodellen im Mittelpunkt des Vorhabens. Doch trotz der vielfältigen Aktivitäten der beteiligten Projekte besteht perspektivisch weiterer Handlungsbedarf. Neben der Weiterentwicklung, Aktualisierung und Optimierung der bereits vorhandenen Maßnahmen (Anrechnungsverfahren, Rahmenordnungen, Handreichungen, Beratungsangebote, Workshops etc.) und der Übertragung erprobter Anrechnungsverfahren auf andere (Weiterbildungs-)Formate besteht auch weiterhin Handlungsbedarf im Hinblick auf die Steigerung von Transparenz und Akzeptanz im Themenbereich „Anrechnung“ (Weichert 2015).

\section{PLAR-Service der Universität Oldenburg}

Obwohl sich die Anrechnungsverfahren hierzulande bislang überwiegend auf formell erworbene Qualifikationen beziehen, stehen inzwischen auch an einigen deutschen Hochschulen unterschiedliche Ansätze und verschiedene praktizierte Verfahren zur Erfassung und Anrechnung außerhalb des formalen Bildungswesens erworbener Lernleistungen zur Verfügung.

Im Januar 2017 startete die Carl von Ossietzky Universität Oldenburg einen zentralen Anrechnungsservice für sämtliche grundständige Bachelor- und konsekutiven Master-Studiengänge, den sogenannten PLAR-Service. Dieser orientiert sich am international verbreiteten Ansatz des „Prior Learning Assessment and Recognition (PLAR)“ (Spencer 2005). Die Einrichtung des Angebots wird durch das vom Europäischen Sozialfonds sowie vom Niedersächsischen Ministerium für Wissenschaft und Kultur (MWK) geförderte Projekt „PLARnet“ unterstützt. Studierende haben durch den PLAR-Service die Möglichkeit, sich informell und non-formal erworbene Kompetenzen auf Grundlage eines Portfolios anrechnen zu lassen. Das Portfolio verwendet u.a. authentische Dokumente (sogenannte „Artefakte“) zum Nachweis informell erworbener Kompetenzen. Im ersten Jahr des Bestehens konnten rund 50 Anrechnungsvorgänge erfolgreich abgeschlossen werden.

PLAR kann als eine spezifische Form der individuellen Anrechnung verstanden werden (Müskens 2006). Der PLAR-Ansatz unterscheidet sich u. a. aufgrund seiner Fokussierung auf individuelle Kompetenzen von anderen Formen der Anrechnung. So versteht Conrad (2006) PLAR als einen Teilbereich des umfassenderen Begriffs RPL (Recognition of Prior Learning):

Prior learning assessment and recognition is itself an arm of the larger umbrella term, recognizing prior learning (RPL). Under the aegis of the latter is contained, in addition to PLAR, the related (but different) processes of credit transfer (Conrad 2006, S. 2).

In einer späteren Veröffentlichung differenziert Conrad (2008, S. 91) ihre Taxonomie der Anrechnung weiter aus und beschreibt RPL als Oberbegriff für PLAR, Credit Transfer und Qualification Recognition. Hier wird deutlich, dass PLAR als ein kompetenzbasierter Ansatz verstanden wird, der insbesondere auch informelles Ler- 
nen einschließt, während in Abgrenzung dazu QR (Qualification Recognition) eine auf Abschlüsse (Qualifikationen) basierte Anrechnung meint. CT (Credit Transfer) entspricht dem deutschen Begriff der „Anerkennung“ hochschulischer Vorleistungen, d. h. bereits erworbener Credit-Punkte.

Die Unterscheidung von PLAR und QR (als zwei mögliche Formen der Anrechnung beruflicher Kompetenzen) wird deutlicher, wenn man betrachtet, welche Formen der Nachweise jeweils Verwendung finden. So basiert QR als qualifikationsorientierte Anrechnungsform meist auf den Curricula (Rahmenstoffpläne, Lernziele etc.) der anzurechnenden beruflichen Qualifikationen. Auch wenn die Anrechnungsentscheidung individuell (für eine oder einen Studierenden) getroffen wird, ist das Ergebnis dieser Entscheidung somit prinzipiell pauschalisierbar: Für eine andere Person, die die gleiche Qualifikation erfolgreich abgeschlossen hat, kommt das Verfahren zu exakt demselben Anrechnungsergebnis.

PLAR hingegen kommt selbst bei Studierenden, die die gleichen Aus- oder Weiterbildungen besucht haben, häufig zu unterschiedlichen Anrechnungsentscheidungen. Hier bilden weniger die Lernergebnisse, die von allen Lernenden erreicht werden, die Grundlage der Anrechnung, sondern vielmehr individuelle Lernergebnisse, die z.B. in Projektarbeiten, Referaten oder einer Anwendung des Gelernten in der Berufspraxis zum Ausdruck kommen.

Anders als bei QR liegen PLAR daher individuelle Fähigkeitsnachweise zugrunde, die üblicherweise in einem Portfolio gesammelt werden. Der Begriff PLAR ist so eng mit Portfolios verbunden, dass PLAR zuweilen mit portfoliobasierter Anrechnung gleichgesetzt wird. So beklagt Conrad (2008): „There is also a tendency in Canada for PLAR to be understood synonymously with the use of portfolios as an assessment methology“ (ebd., S. 91).

Tatsächlich basiert auch das an der Universität Oldenburg verwendete PLARVerfahren ausschließlich auf Portfolios. Innerhalb des Portfolios kann jedoch eine Vielzahl unterschiedlicher Kompetenznachweise Verwendung finden, wie Conrad (2013) verdeutlicht:

The many parts of the portfolio may include a learning narrative, a resume, a statement of educational or career goals, program information, and some type of written demonstration of learning, usually written in response to stated criteria which often take the form of learning outcome at program or course level (ebd., S. 44).

Die Vielfalt möglicher Nachweise erlaubt es, dass PLAR sich auf Kenntnisse, Fähigkeiten und Kompetenzen beziehen kann, die in formellen, non-formalen und/ oder informellen Lernkontexten erworben wurden. Kennepohl (2016) beschreibt dies als ein zentrales Merkmal von PLAR: „The underlying philosophy of PLAR is that it should not matter where or how learning has occurred, but that it has occurred“" (ebd., S. 155).

Eine Besonderheit des PLAR-Ansatzes bilden die in den Portfolios gesammelten Nachweise. Neben formellen Zeugnissen und Zertifikaten kann es sich hierbei auch um authentische Nachweise handeln. Dies sind beliebige Dokumente (oder multimediale Materialien), die aus dem Lern- oder Arbeitsprozess des bzw. der Lernenden 
stammen und die geeignet sind, die anzurechnenden Kenntnisse, Fähigkeiten bzw. Kompetenzen nachzuweisen.

Die Zusammenstellung bzw. Generierung solcher Kompetenznachweise bildet dabei eine nicht unerhebliche Anforderung an die Studierenden. Anders als QR und CT verlangt PLAR von den Lernenden demzufolge eine aktive Mitwirkung am Anrechnungsprozess.

An der Universität Oldenburg wurde daher im Januar 2017 mit dem PLARService eine Stelle eingerichtet, die Studierende aller Fakultäten bei der Erstellung systematischer Anrechnungsportfolios unterstützt. Dabei steht eine Anleitung zur Reflexion der eigenen außerhochschulisch erworbenen Kompetenzen im Vordergrund. In mehreren Beratungsgesprächen wird die Bildungs- und Arbeitsbiografie der Lernenden aufgearbeitet und mit den anzurechnenden Studienmodulen in Verbindung gesetzt.

Der PLAR-Service arbeitet in enger Kooperation mit dem akademischen Prüfungsamt und dem Centrum für lebenslanges Lernen. Durch die hochschulweite Vernetzung des PLAR-Services konnte ein einheitlicher Bearbeitungsprozess für sämtliche Anrechnungsanträge etabliert werden (Abb. 1). Die Entscheidung über

Abb. 1 Ablauf des Anrechnungsprozesses an der Universität Oldenburg

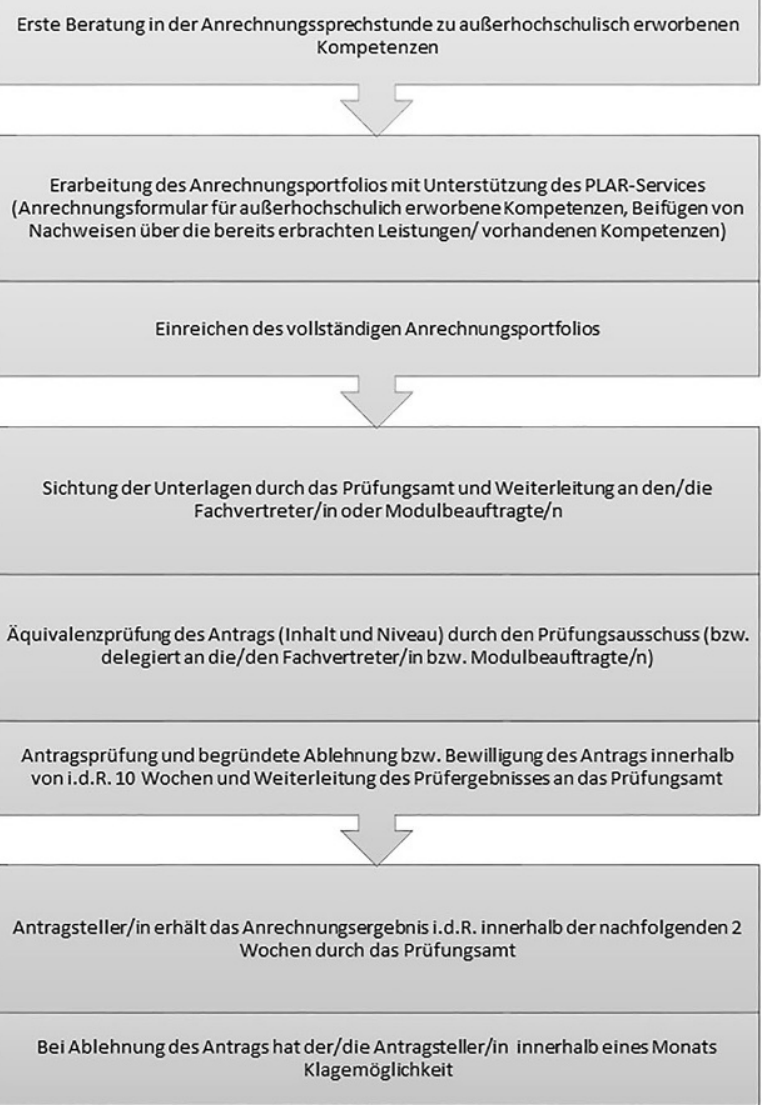


die Anrechnung wird von Fachvertreterinnen und Fachvertretern in den jeweiligen Fakultäten getroffen.

\section{Erfahrungen mit dem PLAR-Service}

Der PLAR-Service wurde von den Studierenden der Universität sehr positiv aufgenommen. Alle bearbeiteten Portfolios bezogen sich (zumindest teilweise) auch auf informelles Lernen. In aller Regel wurden in beruflichen Tätigkeiten erworbene Kompetenzen nachgewiesen, die hinsichtlich ihres Niveaus den Lernergebnissen der entsprechenden Studienmodule entsprachen.

Von zentraler Bedeutung für die Anrechnung informellen Lernens auf Studienmodule erwies sich die Verwendung authentischer Nachweise. Häufig lassen sich die Lernergebnisse informellen Lernens nicht durch formelle Zertifikate oder Zeugnisse nachweisen, so dass ein Nachweis über Dokumente aus dem Lern- oder Arbeitsprozess erforderlich wird.

Schwierigkeiten bereitete vielen Lernenden die Zuordnung ihrer eigenen Kenntnisse und Fähigkeiten zu den Lernergebnissen der Studienmodule, auf die angerechnet werden sollte. Auch die für die Lernenden ungewohnte Form eines Nachweises von Fähigkeiten über authentische Nachweise erforderte eine intensive Beratung durch die Mitarbeiterin des PLAR-Services. Das Gelingen des PLAR-Ansatzes ist eng mit der (pädagogischen) Kompetenz der Beraterinnen und Berater verbunden. Ein entsprechend geschultes Personal bildet daher eine Grundvoraussetzung für den Erfolg portfoliobasierter Anrechnungsverfahren.

\section{Fazit}

Die Möglichkeiten der Anrechnung non-formal oder informell erworbener Kompetenzen werden auch zukünftig eine bedeutsame Rolle im Hochschulbereich einnehmen. Durch die Validierung von Kompetenzen, die im beruflichen Kontext oder auch außerhalb formaler Bildungswege erworben wurden, können auch Personen ohne den erforderlichen Schulabschluss einen Zugang zum Studium erhalten. Zudem können den Studierenden bestimmte Studieninhalte durch den Nachweis äquivalenter Lernleistungen und -erfahrungen erlassen werden, wodurch eine zeitliche Verkürzung des Studiums sowie eine Verringerung der Arbeitsbelastung innerhalb der Studienzeit ermöglicht werden kann (Käpplinger 2003; DAbeKom 2016).

Trotz der dargestellten Problematiken und Unklarheiten hinsichtlich einheitlicher Konzepte und Verfahrenswege für die praktische Ausgestaltung der Identifizierung, Bewertung und Anrechnung informellen Lernens wird die Berücksichtigung, Einbeziehung und Anrechnung formal, non-formal und informell erworbener Lernerfahrungen und Qualifikationen insbesondere vor dem Hintergrund des lebenslangen Lernens und im Kontext der geforderten Durchlässigkeit zwischen den verschiedenen Bildungswegen in Deutschland weiterhin an Aufmerksamkeit gewinnen (Reimer 2006; AK DQR 2011; DAbeKom 2016). 
Open Access Dieser Artikel wird unter der Creative Commons Namensnennung 4.0 International Lizenz (http://creativecommons.org/licenses/by/4.0/deed.de) veröffentlicht, welche die Nutzung, Vervielfältigung, Bearbeitung, Verbreitung und Wiedergabe in jeglichem Medium und Format erlaubt, sofern Sie den/die ursprünglichen Autor(en) und die Quelle ordnungsgemäß nennen, einen Link zur Creative Commons Lizenz beifügen und angeben, ob Änderungen vorgenommen wurden.

\section{Literatur}

AK DQR (2011). Deutscher Qualifikationsrahmen für lebenslanges Lernen. Verabschiedet vom Arbeitskreis Deutscher Qualifikationsrahmen am 22. März 2011. http://www.akkreditierungsrat.de/ fileadmin/Seiteninhalte/Sonstige/BMBF_DQR_aktuell.pdf. Zugegriffen: 17. Mai 2018.

ANKOM (2016). Übergänge von der beruflichen in die hochschulische Weiterbildung. http://ankom.his. de/bmbf. Zugegriffen: 17. Mai 2018.

ANKOM. (2010). Anrechnungsleitlinie - Leitlinie für die Qualitätssicherung von Verfahren zur Anrechnung beruflicher und außerhochschulisch erworbener Kompetenzen auf Hochschulstudiengänge. Wissenschaftliche Begleitung der BMBF-Initiative „Anrechnung beruflicher und außerhochschulisch erworbener Kompetenzen auf Hochschulstudiengänge (ANKOM)“. Hannover/Berlin: VDI/VDE. http://ankom.his.de/know_how/anrechnung/pdf_archiv/ANKOM_Leitlinie_1_2010.pdf. Zugegriffen: 17. Mai 2018.

Annen, S. (2012). Anerkennung von Kompetenzen - Kriterienorientierte Analyse ausgewählter Verfahren in Europa. Bielefeld: wbv.

Björnavåld, J. (1997). Ermittlung und Validierung von früher bzw. nicht formell erworbenen Kenntnissen. Thessaloniki: CEDEFOP.

BMBF - Bundesministerium für Bildung und Forschung (2016). Bund-Länder-Wettbewerb „Aufstieg durch Bildung: offene Hochschulen“. http://www.wettbewerb-offene-hochschulen-bmbf.de/bundlaender-wettbewerb-aufstieg-durch-bildung-offene-hochschulen. Zugegriffen: 17. Mai 2018.

$\mathrm{BMBF}$ - Bundesministerium für Bildung und Forschung (2018). Validierung non-formalen und informellen Lernens in Deutschland. Bericht zur Umsetzung der Empfehlung des Rates vom 20. Dezember 2012 zur Validierung nichtformalen und informellen Lernens. https://www. validierungsverfahren.de/index.php?eID=tx_securedownloads\&u=0\&g=0\&t=1534945271\& hash=cd93973ec56963442283930a388777732d3d0636\&file=/fileadmin/user_upload/valikom/ download/BMBF-Bericht_Validierung_non-formalen_und_infromellen_Lernens_in_Deutschland. pdf. Zugegriffen: 21. Aug. 2018.

Campus France (2016). Anerkennung beruflicher Tätigkeiten und ausländischer Diplome. Webseite von Campus France Deutschland. campusfrance.org - Die nationale Agentur für die Förderung des französischen Hochschulwesens im Ausland, die Betreuung von Studierenden und die internationale Mobilität. www.allemagne.campusfrance.org/node/77466. Zugegriffen: 17. Mai 2018.

Colardyn, D. (1994). Certification of Adult Education. In T. Husen \& T. N. Postlethwaite (Hrsg.), International Encyclopaedia of Education (S. 662-665). Oxford: Imprint.

Conrad, D. (2006). E-portfolios as new learning spaces? Portfolios, paradigms, and pedagogy. Paper presented at EDEN-conference, Vienna. http://priorlearning.athabascau.ca/documents/e-portfoliosEDEN-feb-1.doc. Zugegriffen: 17. Mai 2018.

Conrad, D. (2008). Revisiting the recognition of prior learning (RPL): a reflective inquiry into RPL practice in Canada. Canadian Journal of University Continuing Education, 34(2), 89-110.

Conrad, D. (2013). Assessment challenges in open learning: way-finding, fork in the road, or end of the line. Open Praxis, 5(1), 41-47.

DAbeKom (2016). Anrechnungsverfahren. Webseite der Datenbank zur Anrechnung beruflicher Kompetenzen (DAbeKom). www.dabekom.de/dabekom.html?seite=11. Zugegriffen: 17. Mai 2018.

Dohmen, G. (2001). Das informelle Lernen. Die internationale Erschließung einer bisher vernachlässigten Grundform menschlichen Lernens für das lebenslange Lernen aller. Bonn: BMBF.

Gnahs, D. (2003). Zertifizierung informell erworbener Kompetenzen. Report-Literatur- und Forschungsreport Weiterbildung, 26(4), 88-96.

Greco, L. (2002). L'expérience de la France ouvre une nouvelle perspective. Luxemburger Wort, 27. April 2002.

Hanak, H., \& Sturm, N. (2015). Anerkennung und Anrechnung außerhochschulisch erworbener Weiterbildung. Wiesbaden: Springer VS. 
Hanft, A., Brinkmann, K., Gierke, W. B., \& Müskens, W. (2014). Anrechnung außerhochschulischer Kompetenzen in Studiengängen - Studie: AnHoSt „Anrechnungspraxis in Hochschulstudiengängen “. Oldenburg: Carl von Ossietzky Universität.

Hanft, A,. \& Müskens, W. (2003). Prüfungs- und Dokumentationsmethoden als Instrumente der kompetenzorientierten Erfolgskontrolle internetgestützten Lernens: Ergebnisse des Projekts ,Lernerfolgskontrolle und Zertifizierung des Lernens im Netz und mit Multimedia unter dem Aspekt der Lernkulturveränderung und Kompetenzentwicklung“. Berlin: Arbeitsgemeinschaft Betriebliche Weiterbildungsforschung e.V., QUEM-Report, 80, 177-254.

Hannken-Illjes, K., \& Lischka, I. (2004). Ansätze zur Systematisierung von Lernleistungen im Rahmen eines Credit-Systems und Lebenslangen Lernens, unter Berücksichtigung der europäischen Perspektive. In I. Stamm-Riemer (Hrsg.), Lebenslanges Lernen. Zur Verknüpfung akademischer und beruflicher Bildung (S. 173-264). Berlin: BWV.

Käpplinger, B. (2003). Anerkennung von Kompetenzen: Definitionen, Kontexte und Praxiserfahrungen in Europa. www.die-bonn.de/esprid/dokumente/doc-2002/kaepplinger02_01.pdf. Zugegriffen: 17. Mai 2018.

Kennepohl, D. K. (2016). Incorporating learning outcomes in transfer credit: the way forward for campus Alberta? Canadian Journal of Higher Education, 46(2), 148-164.

KMK - Kultusministerkonferenz (2002). Anrechnung von außerhalb des Hochschulwesens erworbenen Kenntnissen und Fähigkeiten auf ein Hochschulstudium. Beschluss der Kultusministerkonferenz vom 28.06.2002. www.kmk.org/fileadmin/pdf/PresseUndAktuelles/2003/anrechnung.pdf. Zugegriffen: 17. Mai 2018.

KMK - Kultusministerkonferenz (2008). Anrechnung von außerhalb des Hochschulwesens erworbenen Kenntnissen und Fähigkeiten auf ein Hochschulstudium (II). Beschluss der Kultusministerkonferenz vom 18.09.2008. www.kmk.org/fileadmin/veroeffentlichungen_beschluesse/2008/2008_09_18Anrechnung-Faehigkeiten-Studium-2.pdf. Zugegriffen: 17. Mai 2018.

KMK - Kultusministerkonferenz (2010). Ländergemeinsame Strukturvorgaben für die Akkreditierung von Bachelor- und Masterstudiengängen. Beschluss der Kultusministerkonferenz vom 10.10.2003 i.d.F. vom 04.02.2010. www.kmk.org/fileadmin/Dateien/veroeffentlichungen_beschluesse/2003/2003_10_ 10-Laendergemeinsame-Strukturvorgaben.pdf. Zugegriffen: 28. Apr. 2016.

Müskens, W. (2006). Pauschale und individuelle Anrechnung beruflicher Kompetenzen auf Hochschulstudiengänge - das Oldenburger Modell. Hochschule \& Weiterbildung, 1, 23-30.

Müskens, W. (2007). Accreditation of Prior and Experiential Learning im Hochschulbereich. In A. Hanft (Hrsg.), Modellversuch wissenschaftliche Weiterbildung - Entwicklung von Modellen für Planung, Implementierung, Management und Evaluation von weiterbildenden Studiengängen in vernetzten Strukturen (MaweSt) - Abschlussbericht (S. 34-55). Oldenburg: Carl von Ossietzky Universität.

Perker, H., \& Ward, C. (1994). Identification and accreditation of skills and knowledge acquired through life and work experience. Comparative report of practice in France and the United Kingdom. Berlin: European Centre for the Development of Vocational Training.

Räbinger, J., Pehlke-Milde, J., \& Machocki, S. (2008). Anrechnung beruflicher Aus- und Weiterbildung auf ein Studium - eine Chance für die Gesundheitsfachberufe. In S. Matzick (Hrsg.), Qualifizierung in den Gesundheitsberufen: Herausforderungen und Perspektiven für die wissenschaftliche Weiterbildung (S. 229-237). Weinheim: Juventa.

Rat der Europäischen Union (2012). Empfehlung des Rates vom 20. Dezember 2012 zur Validierung nichtformalen und informellen Lernens. Amtsblatt der Europäischen Union (2012/C 398/01). http://eurlex.europa.eu/legal-content/DE/TXT/?uri=CELEX:32012H1222(01). Zugegriffen: 17. Mai 2018.

Reimer, M. (2006). Das ProfilPASS-System zur Ermittlung und Dokumentation informell erworbener Kompetenzen. Zeitschrift für Bildungsverwaltung, 22(1/2), 21-27.

Schreiber, D. (2009). Vorwort. In: M. Linten \& S. Prüstel (2009): Auswahlbibliografie „Zertifizierung und Anerkennung informell erworbener Kompetenzen“. (S. 3-4). https://www.bibb.de/dokumente/ pdf/a1bud_auswahlbibliografie-zertifizierung.pdf. Zugegriffen: 17. Mai 2018.

Spencer, B. (2005). Defining prior learning assessment and recognition. In L. M. English (Hrsg.), Encyclopaedia of adult education (S. 508-512). London: Palgrave Macmillan.

Stamm-Riemer, I., Loroff, C., Minks, K.-H., \& Freitag, W. (Hrsg.). (2008). Die Entwicklung von Anrechnungsmodellen - Zu Äquivalenzpotenzialen von beruflicher und hochschulischer Bildung. www. dzhw.eu/pdf/pub_fh/fh-200813.pdf. Zugegriffen: 17. Mai 2018.

Sturm, N., \& Hanak, H. (2018). Identifizierung der Kernprozesse in Anrechnungsverfahren: eine Voraussetzung für die nachhaltige Implementierung in Hochschulstrukturen. In T.C. Feld \& S. Lauber-Pohle (Hrsg.), Organisation und Profession - Felder erwachsenenpädagogischer Forschung. Wiesbaden: Springer VS. 
Triby, E. (2009). Accreditation of prior experiential learning and the development of higher education. European Journal of Vocational Training, 46(1), 114-128.

Weichert, D. (2015). Handreichung Anrechnung, Teil 2 - Ein Einblick in die Praxis. Handreichung der wissenschaftlichen Begleitung des Bund-Länder-Wettbewerbs „Aufstieg durch Bildung: offene Hochschulen“. Berlin: BMBF.

Werquin, P. (2015). Anerkennung informell erworbener beruflicher Kompetenzen. www.giz.de/ fachexpertise/html/12702.html. Zugegriffen: 17. Mai 2018.

Zürcher, R. (2007). Informelles Lernen und der Erwerb von Kompetenzen - Theoretische, didaktische und politische Aspekte. https://erwachsenenbildung.at/downloads/service/nr2_2007_informelles_lernen. pdf. Zugegriffen: 17. Mai 2018. 